\title{
Novel Resonant-Tunneling Multiple-Threshold Logic Circuit Based on Switching Sequence Detection
}

\author{
Takao Waho, Kazufumi Hattori, and Kouji Honda \\ Department of Electrical and Electronics Engineering \\ Faculty of Science and Engineering \\ Sophia University \\ 7-1 Kioicho, Chiyoda-ku, Tokyo 102-8554, Japan \\ waho@sscd.ee.sophia.ac.jp
}

\begin{abstract}
We present a novel multiple-threshold circuit using resonant-tunneling diodes (RTDs). The logic operation is based on detecting a switching sequence in the RTD circuit. This scheme enables us to increase the number of threshold voltages by more than a factor of two compared with previous RTD-based MVL circuits. SPICE simulation shows that the circuit can operate at a clock frequency as high as $10 \mathrm{GHz}$. A 4-bit flash analog-to-digital converter, which uses the present circuit as a quantizer, is also discussed.
\end{abstract}

\section{Introduction}

Resonant-tunneling diodes (RTDs) connected in series exhibit a multiple-peak structure in their current-voltage characteristics. This provides a unique opportunity to design multiple-valued logic (MVL) circuits with simple circuit configuration [1 - 4]. We have developed such RTD-based MVL circuits, which we call monostable-tomultistable transition logic (MML) [5, 6]. As the circuit evolves from a monostable state to a multistable state, various logic operations are obtained. Because of superior dynamic properties of devices used in the implementation, i. e., InGaAs-based RTDs [7] and heterojunction fieldeffect transistors (HFETs), MML circuits operate at a frequency as high as $10 \mathrm{GHz}[8,9]$. An experimental 2-bit 5-GS/s analog-to-digital converter (ADC) is also demonstrated by using the MML gate as a front-end quantizer [10]. Such a GHz-range MVL gate is expected to be a key circuit block in a future multi-band, multimode wireless communications systems, where digital signal processing technique is introduced not only in the baseband region but also in the intermediate and radio frequency (IF and RF) domains [11].

In this paper, we propose a novel MML circuit that considerably extends the capability of implementing logic functions, while keeping the operation speed in a $\mathrm{GHz}$ range. The gate operation is obtained by detecting a switching sequence in series-connected RTDs that arises as the applied voltage increases. This is in contrast to the previous MML gate, where only the final state after the switching sequence was used in the circuit operation. Since there are several switching sequences that result in the same final state, the present gate can provide more logic functions than the previous ones by distinguishing one sequence from others. In particular, we can increase the number of threshold voltages by more than a factor of two. If this gate is used as a quantizer of flash analog-todigital converters (ADCs), the number of comparators can be much reduced, which would be a great advantage over conventional ultrahigh-speed ADCs.

In Section 2, the operation principle of a present MML gate is described in comparison with a conventional one. Then the circuit configuration is presented. In Section 3, $10-\mathrm{GHz}$ SPICE simulation results are demonstrated. Finally, a 4-bit flash analog-to-digital converter is discussed as a possible application in Section 4.

\section{Switching Sequence Detection}

In this section, we will briefly review a previous MML circuit. Next, we will describe operation principle and configuration of the present circuit. Finally, the number of threshold voltages will be compared with that in the conventional scheme. 


\subsection{Previous MML circuit [5, 6]}

A previous MML circuit consisted of series-connected RTDs and a heterojunction FET (HFET), as shown in Fig. 1(a). The gate operation resulted from the sequential switching of RTDs at the rising edge of the clocked voltage applied to the CK terminal. The high level of the clock was adjusted so that two RTDs out of the four, A, B, $\mathrm{X}$ and $\mathrm{Y}$, switched from the low-resistive on-state to the high-resistive off-state. Under this condition, there are three final states that can be represented by a pair of switched-off RTDs. Those are (X, Y), (B, Y) and (A, B), if the peak current of the RTDs is designed as shown in Fig. 1(b). Here, we included the HFET drain current as a part of the peak currents of X and Y. These "effective" peak currents increase as the input voltage increases, though the actual peak currents of $\mathrm{X}$ and $\mathrm{Y}$ are constant as those of $\mathrm{A}$ and $\mathrm{B}$.

As we increase the voltage applied to the CK terminal in Fig. 1(a), the RTD with the smallest peak current switches first, followed by the RTD with the second smallest peak current, and so forth. This results in the RTD sequential switching that is of particular interest here. If the input voltage $V_{\text {in }}$ is in region (i) in Fig. 1(b), Y switches first followed by $\mathrm{X}$, and the final state is $(\mathrm{X}, \mathrm{Y})$. Since the RTD voltage increases when it switches from the on- to off-state, the output corresponding to $(\mathrm{X}, \mathrm{Y})$ is the highest. Similarly, the final state is $(B, Y)$ if the input is in (ii), and it is (A, B) if the input is in (iii). This results in the ternary complement output, which decreases from the highest through the middle to the lowest as shown in Fig. 1(c). The threshold voltages, $V_{10}$ and $V_{20}$, are determined by the intersections (the solid circles in Fig. 1(b)) in a diagram indicating the peak current as a function of the input voltage $V_{\text {in }}$. (a)

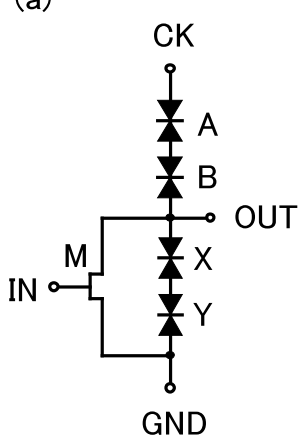

(b)

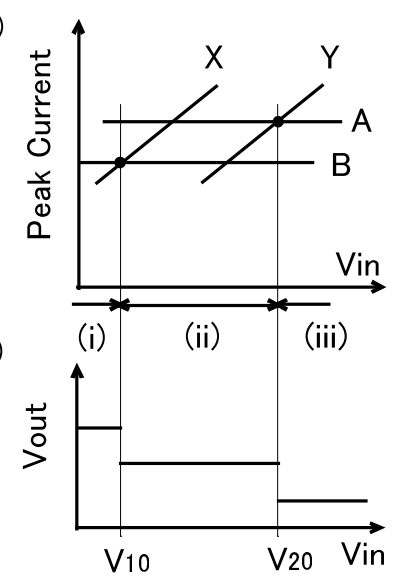

Figure 1. Monostable-to-multistable transition logic gate (MML) previously proposed. Circuit configuration (a), peak current as a function of input voltage (b), and voltage transfer characteristic (c).

\subsection{Present MML circuit}

In the previous MML circuits, the two threshold voltages, $V_{10}$ and $V_{20}$, were obtained as two intersections in the peak current diagram (Fig. 1(b)). There are two other intersections that are left unused. If we can use these for implementing logic functions, we can double the number of thresholds from two to four. Fig. 2(a) shows these two points by open circles, as well as four threshold voltages, $V_{1}, \quad V_{2}, \quad V_{3}$ and $V_{4}$. As presented below, we have accomplished a new mechanism to make use of these four threshold voltages.

The switching sequence in series-connected RTDs is dominated by the peak current, $I_{\mathrm{p}}$, as mentioned above. At an intersection in the $I_{\mathrm{p}}-V_{\text {in }}$ diagram, the relation between two peak currents changes, so does the switching sequence. For example, when $V_{\text {in }}=V_{1}$, the relation between the peak currents of $\mathrm{X}$ and $\mathrm{B}$ changes. For $V_{\text {in }}<$ $V_{1}$ (region (i) in Fig. 2(a)), the peak current of $\mathrm{X}, I_{\mathrm{p}}(\mathrm{X})$, is smaller than $I_{\mathrm{p}}(\mathrm{B})$, while $I_{\mathrm{p}}(\mathrm{B})<I_{\mathrm{p}}(\mathrm{X})$ for $V_{\text {in }}>V_{1}$ (region (ii)). In region (i), therefore, $\mathrm{X}$ switches earlier than $\mathrm{B}$, while in region (ii), B switches earlier than X. The RTD switching sequences for the whole input voltage ranges can be determined in this manner, and are shown in Fig. 2(b). By distinguishing a switching sequence from others, (a)

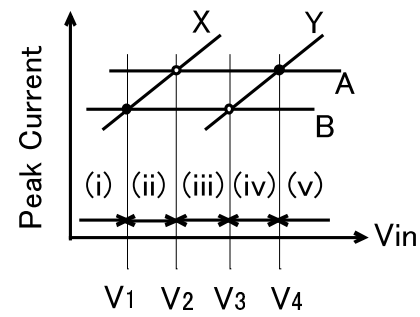

(b)

$\begin{array}{ll} & \text { Switching Seq. } \\ \text { (i) } & \text { Y-X-B-A } \\ \text { (ii) } & \text { Y-B-X-A } \\ \text { (iii) } & \text { Y-B-A-X } \\ \text { (iv) } & \text { B-Y-A-X } \\ \text { (v) } & \text { B-A-Y-X }\end{array}$

Figure 2: Basic idea of the novel logic gate. Peak current as a function of the input voltage showing four threshold voltages, $V_{1}$ through $V_{4}$ (a) and RTD switching sequence in each input region (b).

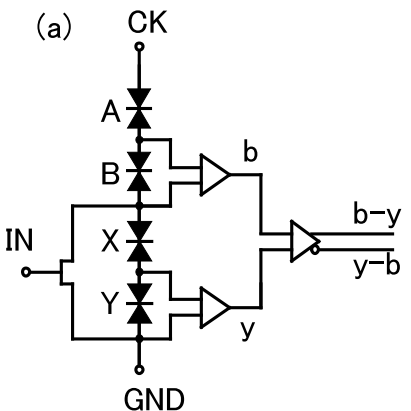

(b)

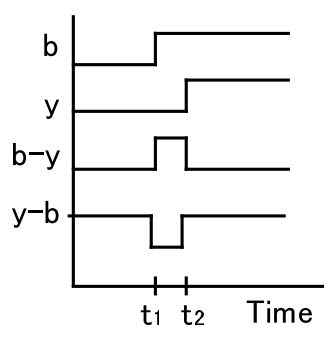

Figure 3: Detection of RTD switching sequence. Detection circuit (a) and output waveforms (b). Here, RTD B switches at $t_{1}$, followed by $Y$ at $t_{2}$. 
therefore, we can obtain the gate operation with the four threshold voltages of $V_{1}, V_{2}, V_{3}$ and $V_{4}$, instead of two, $V_{10}$ and $V_{20}$, in the previous circuit.

We can identify whether or not an RTD switches by measuring the voltage difference between the RTD terminals, because the voltage difference increases abruptly when the RTD switches. Therefore, we have to measure the RTD voltages, and compare them with each other to detect the switching sequence. By using

(a)

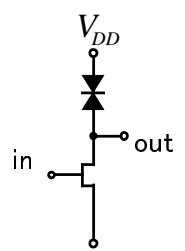

(b)

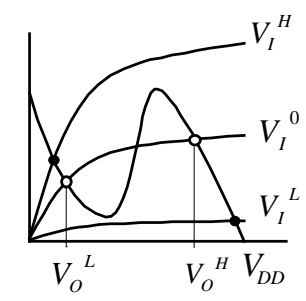

(c)

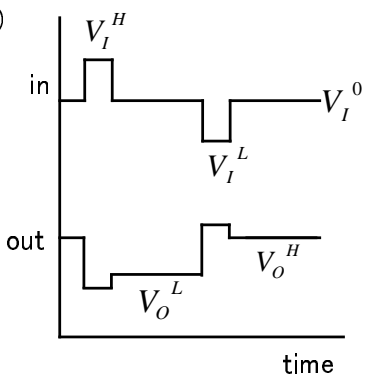

Figure 4. RTD/HFET latch. Circuit configuration (a), output characteristics (b), and schematic waveforms showing latch operation (c).

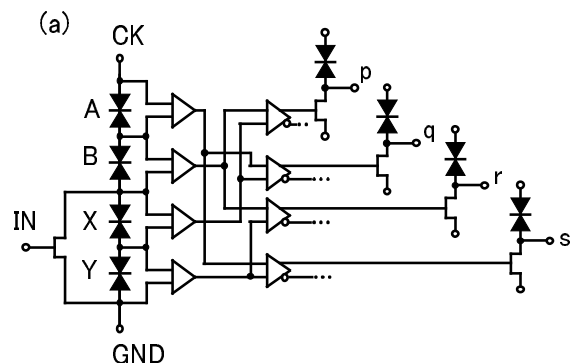

(b)
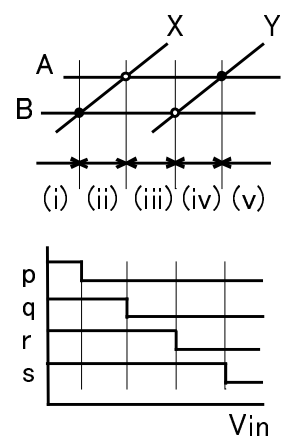

Figure 5. Present multiple-threshold circuit based on RTD switching sequence detection. Circuit configuration (a) and schematic output waveforms (b). differential amplifiers, we have built a new MML gate that recognizes the switching sequence (Fig. 3). If RTD B switches off at $t_{1}$ and RTD Y follows at $t_{2}\left(>t_{1}\right)$, the differential output b-y is a positive pulse. A simple latch consisting of an HFET with an RTD load is used to hold the comparison result. As shown in Fig. 4, a positive pulse results in a low output voltage $V_{\mathrm{O}}{ }^{L}$, while a negative pulse results in a high output voltage $V_{O}{ }^{H}$. In this manner, even though the final circuit states are the same (all RTDs switch off), we can distinguish one particular path from others by measuring the latch outputs.

Figure 5 describes a complete MML circuit. Each RTD is followed by a differential amplifier to detect whether or not the RTD switches, and the outputs are fed into the second differential amplifiers that compare the output voltages from two RTDs. If the RTD, which is connected to the positive input terminal of the second amplifier, switches earlier (later) than the RTD connected to the negative terminal, the output of the second amplifier is a positive (negative) pulse. Four RTD/HFET latches following the second amplifiers thus hold the comparison result. The output waveforms corresponding to each region from (i) to (v) are shown in Fig. 5(b). It should be noted that the complementary signal is also available from the differential amplifiers. We will use the complementary

Table 1. Number of Thresholds

\begin{tabular}{|c|c|c|c|c|c|c|}
\hline \multirow{2}{*}{} & \multicolumn{7}{|c|}{ RTD Count } \\
\cline { 2 - 7 } & 2 & 4 & 6 & 8 & $\ldots$ & $2 N$ \\
\hline $\begin{array}{c}\text { Present MML } \\
\text { (Fig. 5) }\end{array}$ & 1 & 4 & 9 & 16 & $\ldots$ & $N^{2}$ \\
\hline $\begin{array}{c}\text { Modified MML } \\
\text { (Fig. 6) }\end{array}$ & 1 & 6 & 15 & 28 & & $N(2 N-1)$ \\
\hline $\begin{array}{c}\text { Previous MML } \\
\text { (Fig. 1) }\end{array}$ & 1 & 2 & 3 & 4 & $\ldots$ & $N$ \\
\hline
\end{tabular}

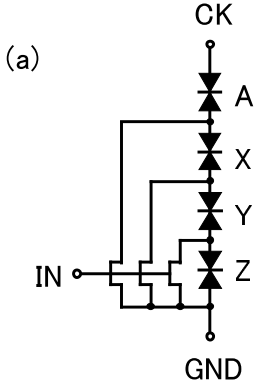

(b)

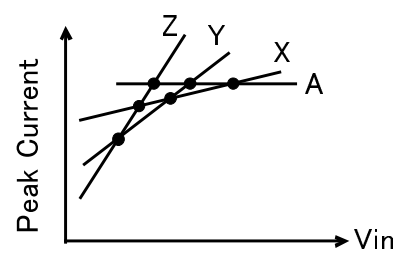

Figure 6. Gate with the maximized number of thresholds. Circuit configuration (a) and a diagram showing a relation between peak current vs. input voltage (b). Closed circles indicate six thresholds. 
output to synthesize a literal function in the next section.

By using the new scheme described above, we can increase the number of threshold voltages while keeping the number of RTDs unchanged. Table I compares the number of thresholds for the present and previous MML gates. A present gate, consisting of $2 N$ RTDs connected in series, has $N^{2}$ threshold voltages, while a previous one has $N$ thresholds. More thresholds can be obtained if we adopt the circuit configuration shown in Fig. 6(a). Here, two additional HFETs are attached to obtain different slopes in the peak-current diagram shown in Fig. 6(b). The number of the intersections between the four lines was maximized, so does the number of threshold voltages as shown in Table I. (a)<smiles>[2H]C1CC2C=C(/C=C\[B])C1C2</smiles>

(b)

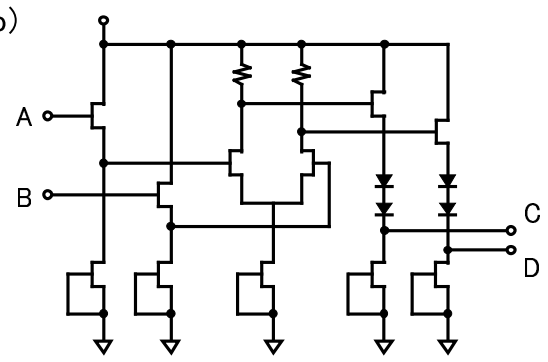

Figure 7. Differential amplifier using a sourcecoupled pair. Symbol (a) and circuit configuration (b).

\section{Simulation Results}

In this section, we will describe SPICE simulation results indicating a possible gate operation at a clock frequency of $10 \mathrm{GHz}$.

The differential amplifier for detecting the switching sequence was designed with a source-coupled pair as shown in Fig. 7. The SPICE simulation was performed for the circuit depicted in Fig. 3 to examine the logic operation as well as to estimate the operation speed. Figure 8 shows the results for two input voltages: $V_{\text {in }}=$ $0.12 \mathrm{~V}$ (a) and $0.3 \mathrm{~V}$ (b). The former corresponds to the switching sequence of $\mathrm{Y} \rightarrow \mathrm{B}$, which means $\mathrm{Y}$ switches first followed by B, while the latter corresponds to B $\rightarrow$ Y. At $0 \mathrm{ps}$, the clock voltage supplied to the $\mathrm{CK}$ terminal in Fig. 3(a) was zero. It started to increase linearly at $20 \mathrm{ps,}$ reached to $5.5 \mathrm{~V}$ at $70 \mathrm{ps}$, and stayed at this value until $120 \mathrm{ps}$. The voltage was measured from the GND terminal. The value of $5.5 \mathrm{~V}$ was chosen because this was large enough for all the RTDs to switch off at this voltage. The upper graphs in Fig. 8 show the outputs of the first differential amplifiers in Fig. 3(a), which reflect the difference in the switching times of $\mathrm{B}$ and Y. The middle graphs in Fig. 8 show the outputs of the second amplifier. A negative pulse in (a) and a positive pulse in (b) agree well with expected ones shown in Fig. 3(b).

The two graphs at the bottom depict the output signals from the RTD/HFET latch, which corresponds to " $\mathrm{r}$ " in Fig. 5(a). We optimized the area of the RTD used in the latch so that the latch worked properly for negative and positive pulses. The simulation results agree well with the designed behavior, again, i. e., for a negative pulse the latched value was high, while for a positive one it was low.

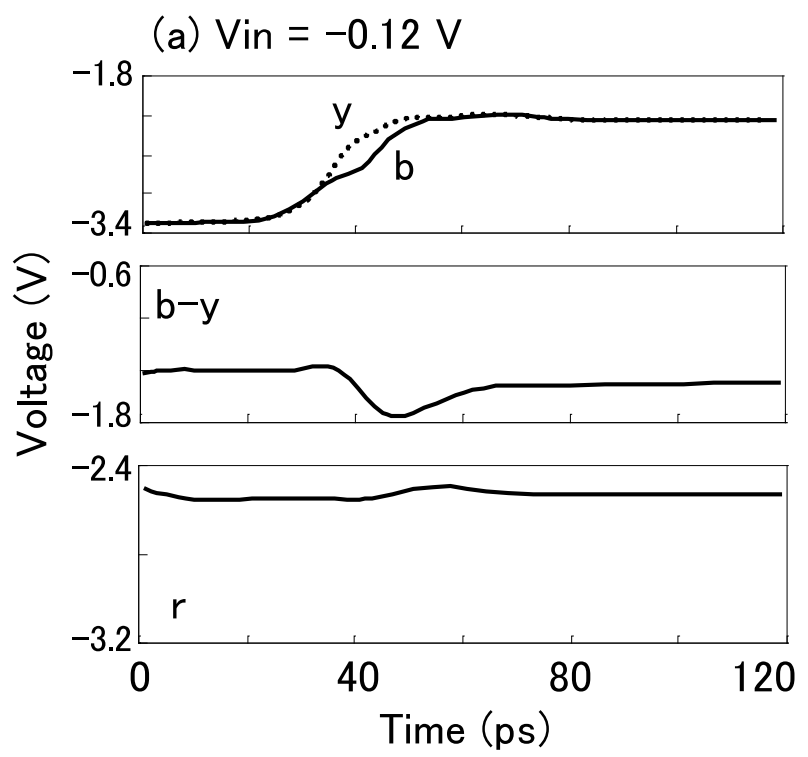

(b) $\operatorname{Vin}=0.3 \mathrm{~V}$
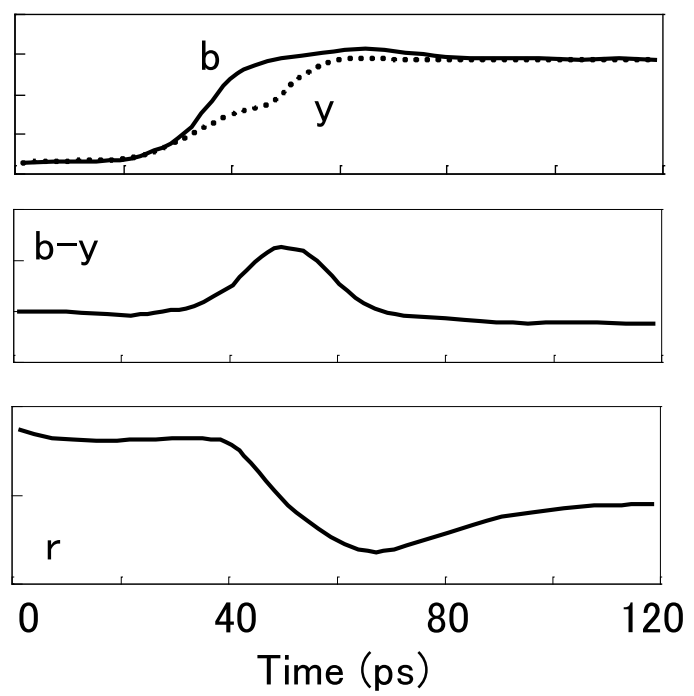

Figure 8. Simulation results calculated for two typical inputs: one corresponds to the case where RTD Y switches followed by RTD B (a), and the other corresponds to the inverted sequence (b). 
The output signals from the latch were almost settled to the final values at $70 \mathrm{ps}$. It should be noted that the clock started rising at 20 ps in Fig. 8 and that the rise time was $50 \mathrm{ps}$. The result indicates, therefore, that the present gate can operate at frequencies as high as $10 \mathrm{GHz}$.

By adding OR/NOR gates to the latch outputs, we can synthesize any one-variable function. We used a source-coupled FET logic (SCFL) OR/NOR gate as shown in Fig. 9. As a simple example, we implemented a literal function by taking AND of $\bar{p}$ (the complement of p) and $r$ (for $p$ and r, see Fig. 5). To use the OR/NOR gate as an AND gate, the negative logic convention was adopted. Simulation results are shown in Fig. 10. The circuit included eight RTDs (four connected in series and

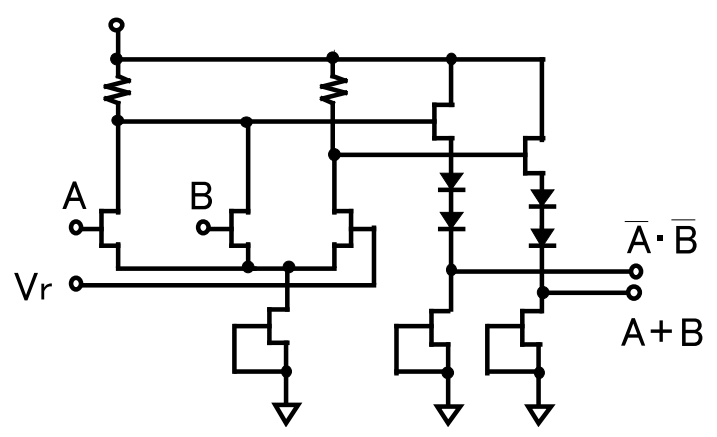

Figure 9. SCFL (source-coupled FET logic) OR/NOR gate.

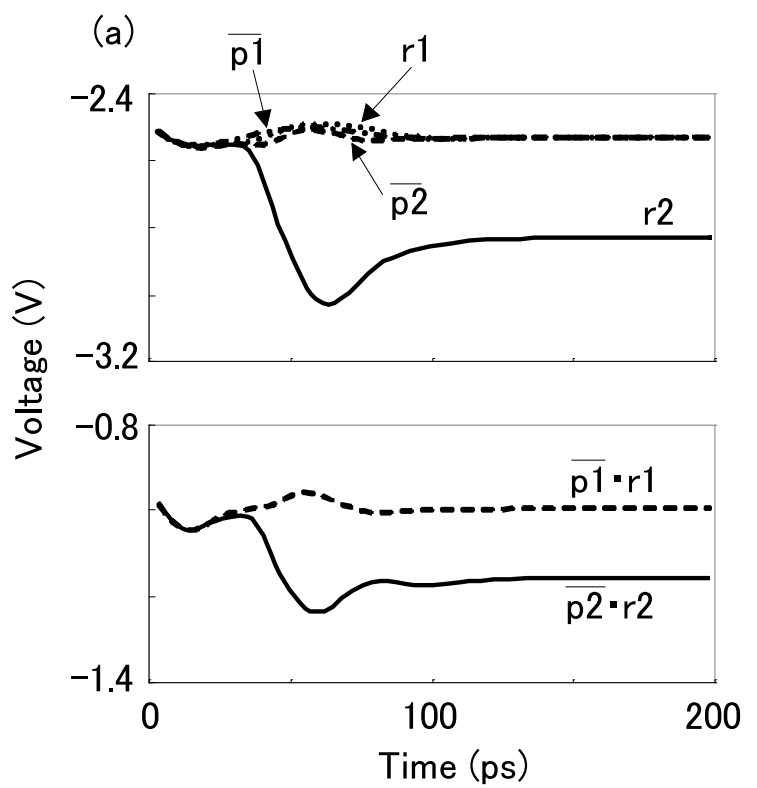

four used in the latch) and 95 HFETs. In the graph on the top in Fig. 10(a), the output signals from the latches are plotted. $\overline{\mathrm{p} 1}$ and $\mathrm{r} 1$ are the latch outputs for $V_{\text {in }}=-0.12 \mathrm{~V}$, while $\overline{\mathrm{p} 2}$ and $\mathrm{r} 2$ are for $V_{\text {in }}=0.3 \mathrm{~V}$. For $\overline{\mathrm{p} 1}$ and $\overline{\mathrm{p} 2}$, the latch input was connected to the complement terminal of the second differential amplifier in Fig 5(a). $\overline{\mathrm{p} 1} \cdot \mathrm{r} 1$ and $\overline{\mathrm{p} 2} \cdot \mathrm{r} 2$ were settled immediately after the change in the latch output. Figure 10(b) shows voltage transfer characteristics of the present literal gate. We plotted $\bar{p} \cdot r$ sampled at $120 \mathrm{ps}$ for input voltages varying from $-0.4 \mathrm{~V}$ to $0.4 \mathrm{~V}$ in $0.02-\mathrm{V}$ steps. Thus, the literal function was successfully demonstrated.

\section{Application to ADC}

By combining literal outputs of various threshold voltages, we can obtain a flash analog-to-digital converter (ADC). In this section, we will briefly discuss a 4-bit flash ADC, which employs the present MML gate as a quantizer.

The block diagram of a 4-bit flash ADC using the present MML gates is shown in Fig. 11. The emitter sizes of RTDs in the MML gates were varied from $8 \mu \mathrm{m}^{2}$ to 18 $\mu \mathrm{m}^{2}$. These sizes were adjusted so that the 16 threshold voltages were uniformly distributed by one leastsignificant-bit interval $(50 \mathrm{mV})$ within the full scale of the input signal (- $0.3 \mathrm{~V}$ to $0.3 \mathrm{~V})$. Four $\mathrm{MML}$ gates are adequate, because it provides four thresholds per gate. The total number of devices in the ADC, including RTDs,

(b)

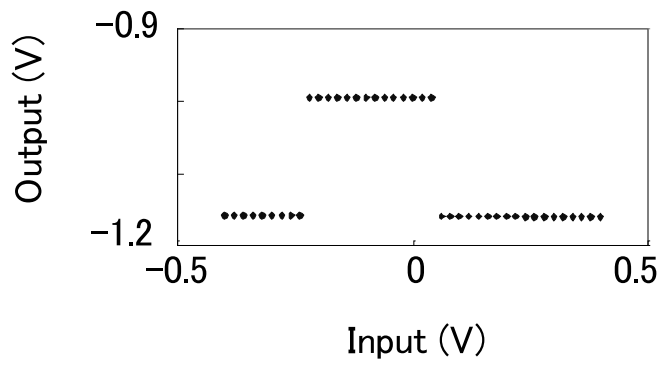

Input (V)

Figure 10. Simulation results for $V_{\text {in }}=-0.12 \mathrm{~V}(\overline{\mathrm{p} 1}$ and $\mathrm{r} 1)$ and $0.3 \mathrm{~V}(\overline{\mathrm{p} 2}$ and $\mathrm{r} 2)(\mathrm{a})$, and voltage transfer characteristic (b). $\overline{\mathrm{p} 1}, \overline{\mathrm{p} 2}, \mathrm{r} 1$ and $\mathrm{r} 2$ are the latch outputs shown in Fig. 5. In (b), the values of $\overline{\mathrm{p}} \cdot \mathrm{r}$ sampled at $120 \mathrm{ps}$ are plotted for various inputs from $-0.4 \mathrm{~V}$ to $0.4 \mathrm{~V}$. 
HFETs, diodes and resistors, was 503. The power dissipation was estimated to be $7.9 \mathrm{~W}$, which comprised $5.5 \mathrm{~W}$ of the RTD quantizer part and $2.4 \mathrm{~W}$ of the encoder circuit. The relatively large quantizer contribution can be reduced by adjusting the current-voltage characteristics of RTDs, in particular the peak voltage $V_{\mathrm{P}}$. In this simulation, we assumed $V_{\mathrm{P}}=0.3 \mathrm{~V}$, which was based on the experimental results. By decreasing $V_{\mathrm{P}}$, we can employ smaller $V_{\mathrm{CK}}$, which would reduce the power dissipation.

Although the present ADC needs more devices than the previous RTD-based ADC [11], it should be noted that the numbers of quantizer RTDs and HFETs (M1 through M4 in Fig. 11) were reduced to 16 and four, respectively. If we construct such an ADC by using the previous 4-RTD MML gate, 32 RTDs and eight HFETs are necessary, because each gate provides only two threshold voltages, instead of four in the present gate (compare Fig. 1 with Fig. 2). This is achieved at a cost of additional circuits following them, such as differential amplifiers. Since the analog front-end block of ultrahigh-speed ADCs dominates its performance, the compact quantizer should be a big advantage. This relaxes the process margin of RTDs and HFETs. In addition, this reduces input capacitance of the ADC considerably, which alleviates the driving capability of a GHz-range pre-amplifier. Hence, it is concluded that the ADC presented here is suitable for ultrahigh-speed operation above a few GHz.

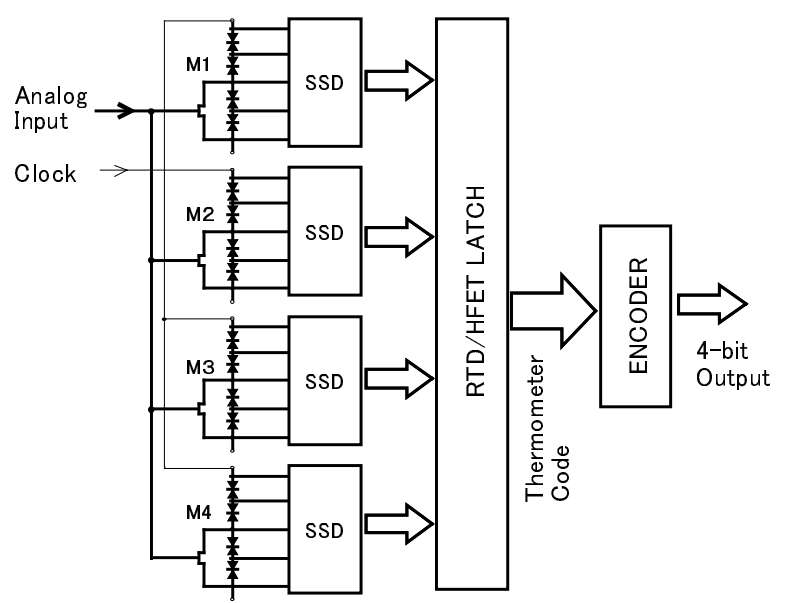

Figure 11. Block diagram of 4-bit flash analog-todigital converter using the present circuits as front-end quantizers. SSD stands for the switching sequence detection circuit.

\section{Conclusion}

Based on a new scheme of detecting the RTD switching sequence, we presented a multiple-threshold logic circuit. This enabled us to increase the number of threshold voltages by more than a factor of two compared with previous RTD-based MVL circuits. SPICE simulation indicated that the circuit operated at a clock frequency as high as $10 \mathrm{GHz}$. A 4-bit flash analog-todigital converter, being suitable for a-few-GS/s operations, was also discussed.

\section{References}

[1] L. J. Micheel, A. H. Taddiken, and A. C. Seabaugh, "Multiple-valued Logic Computation Circuits Using Micro- and Nanoelectronics Devices," Proc. 23 ${ }^{\text {rd }}$ IEEE Int. Symp. MultipleValued Logic, pp. 164 - 169, 1993.

[2] H. C. Lin, "Resonant Tunneling Diodes for Multi-Valued Digital Applications," Proc. 24 $4^{\text {th }}$ IEEE Int. Symp. MultipleValued Logic, pp. 188 - 195, 1994.

[3] P. Mazumder, S. Kulkarni, M. Bhattacharya, J. P. Sun, and G. I. Haddad, "Digital Circuit Applications of Resonant Tunneling Diodes," Proc. IEEE, vol. 86, no. 4, pp. 664 - 686, April 1998.

[4] J. P. A. van der Wagt, H. Tang, T. P. E. Broekaert, A. C. Seabaugh, and Y. -C. Kao, "Multibit Resonant Tunneling Diode SRAM Cell Based on Slew-Rate Addressing," IEEE Trans. Electron Devices, vol. 46, no. 1, pp. 55 - 62, January 1999.

[5] T. Waho, "Resonant Tunneling Transistor and Its Application to Multiple-Valued Logic Circuits," Proc. $25^{\text {th }}$ IEEE Int. Symp. Multiple-Valued Logic, pp.130 - 138, 1995.

[6] T. Waho, K. J. Chen and M. Yamamoto, "ResonantTunneling Diode and HEMT Logic Circuits with Multiple Thresholds and Multi-Level Output," IEEE J. Solid-State Circuits, Vol. 33, No. 2, pp. 268 - 274, February 1998.

[7] N. Shimizu, T. Nagatsuma, T. Waho, M. Shinagawa, M. Yaita and M. Yamamoto, "InGaAs/AlAs Resonant Tunneling Diodes with Switching Time of 1.5 ps," Electron. Lett., vol. 31, no. 19, pp. 1695 - 1697, September 1995.

[8] T. Itoh, T. Waho, K. Maezawa, and M. Yamamoto, "10-GHz Operation of Multiple-Valued Quantizers Using ResonantTunneling Devices," IEICE Trans. Information and Systems, vol. E82-D, no. 5, pp. 949 - 954, May 1999.

[9] T. Itoh, T. Waho, J. Osaka, H. Yokoyama, and M. Yamamoto, "Ultrafast Analog-to-digital Converter Using ResonantTunneling Ternary Quantizers," Digest of IEEE MTT-S Int. Microwave Symp., Baltimore MD, June 1998, pp. 197 - 200.

[10] J. A. Wepman, "Analog-to-Digital Converters and Their Applications in Radio Receivers," IEEE Communication Mag., pp. 39 - 45, May 1995.

[11] T. Waho, T. Itoh, K. Maezawa, and M. Yamamoto, "Multi$\mathrm{GHz}$ A/D Converter Using Resonant-Tunneling Multiple-Valued Logic Circuits," 1998 IEEE ISSCC Digest of Technical Papers, pp. $258-259$. 